\title{
DISTINCTIONS AMONG DIFFERENT TYPES OF GENERALIZING IN INFORMATION SYSTEMS RESEARCH
}

\author{
Richard Baskerville \\ Georgia State University \\ U.S.A. \\ Allen S. Lee
Virginia Commonwealth University
U.S.A.
}

Abstract

It is incorrect and even harmful that many information systems researchers typically criticize their own intensive (qualitative, interpretive, critical, and case) research as lacking "generalizability." We untangle and distinguish the numerous concepts now confounded in the single term "generalizability," which are generality, generalization, generalize, general, and generalizing. These clarified terms allow us to identify four distinct forms of generalizing (everyday inductive generalizing, everyday deductive generalizing, academic inductive generalizing, and academic deductive generalizing), each of which we illustrate with an information systems-related example. The clarified terms provide the basis for an explanation of how information systems researchers who perform intensive research may properly lay claim to generality for their research.

Keywords: Action research, case study, epistemology, experimental research, field study, intensive research, laboratory study, research methodology, statistical methods qualitative research. 
For academic researchers in information systems (IS), the concept of "generalizability" has been developing and maturing with the growing acceptance of intensive research approaches (i.e., qualitative, interpretive, critical, and case research ${ }^{1}$ ). Once deemed a property of statistically based research alone, generalizability has been gaining recognition as an achievable ideal in intensive research as well (Lee 1989). Still, it remains common for authors of published intensive research articles to flagellate themselves in their own "discussion" sections for the lack of generalizability of their findings. There they typically blame this supposed failure on their having examined "only" a single case, or "only" three technologies, or "only" two organizations, or "only" one point in time, and so forth. We believe that such self-flagellation is not necessary (i.e., the completed intensive research can indeed claim "generalizability" if it is properly performed and presented). Such unwarranted self-criticism can even be harmful (i.e., it can reflect and reproduce the hegemony of large-sample statistical research over all other forms of scholarly inquiry). The purpose of this study is to clarify the different processes of generalizing so that academic researchers in IS can better achieve and securely claim "generalizability" (or, as we will rename it, "generality") in their research.

The first section of the paper after this introduction will identify and distinguish different terms now confounded in the single term, "generalizability." The terms that we will unconfound and extract from "generalizability" are generality, generalization, generalize, general, generalizing, and even (after we define it) generalizability itself. In the section after that, we will define four types of generalizing and classify them according to the dimensions of "reasoning process" (inductive $v s$. deductive generalizing) and "context" (inquiry in everyday life $v s$. inquiry in academic research). The same section will illustrate the four types with empirical examples from the published literature. In the third section, we discuss an appropriate way in which information systems researchers may indeed lay claim to generality for their research. Then, in the last section, we will bring out the ramifications of the four distinct forms of generalizing for current and future research practices in IS. There we will indict and dismiss the dysfunctionality of self-flagellation for the often imagined sin of "lack of generalizability" in intensive research, as well as proclaim our emancipation to a research environment with a better (or "generalized," as it were) conception of "generalizability."

\section{Unconfounding and Renaming "Generalizability"}

In the way that academic researchers now use the term "generalizability," it regards the extent to which an academic researcher's theory does or does not apply to empirical referents (i.e., real-world situations) apart from the one that the researcher examined in his or her study. A case researcher, for example, might offer the self criticism (in the discussion section of her published case study) that the theory she developed "lacks

'We take this characterization of "intensive research" from M. Lynne Markus and Allen S. Lee's call for papers for a special issue of MIS Quarterly on intensive research. They, in turn, took the term "intensive research" from Karl Weick and used it to refer to the diversity of forms of empirical information systems research falling outside of the quantitative and positivist genre, including qualitative positivist (and nonpositivist) research, interpretive research, critical social theory research, and case study research. 
generalizability" because she based it on observations of only one information technology in only one organization. However, whether or not we would agree with her self criticism, we believe that she means generality, not generalizability.

In this study, we use the term generalizability more specifically. We distinguish it from generality, generalization, generalize, general, and generalizing. We define generalizability to refer to a theory's potential (as the suffix in generalizability signifies) to come to possess the quality of generality. Generality refers to the range of phenomena across which the theory has been demonstrated to hold. Here, generality is the outcome or product of the process of generalizing a theory that, initially, was able to be generalized (i.e., generalizable). To generalize is to engage in the process of generalizing. A theory of perfect generality would apply to the entire class of empirical referents that it purports to explain; a theory with less generality would apply only to a subset of this class. ${ }^{2}$ In other words, we identify generalizability as a property of a theory at the beginning of an empirical investigation, and generality as a property of a theory at the end of the investigation (where the results of the investigation are favorable). The resulting generalization, in this scheme, would be the theory in the form in which it emerges from the empirical investigation. Finally, a theory possessing generality can also be described with the adjective general.

Methodologically speaking, generalizability is a potential that a theory has, just as falsifiability is a potential that a theory has. Just as (1) a researcher can take a newly formulated and untested theory that has the property of falsifiability (i.e., it is falsifiable, but has not yet been falsified) and then (2) the researcher, in examining the theory empirically, can falsify it and thereby demonstrate its falsity, we can say that (1) a researcher can take a newly formulated and untested theory that has the property of generalizability (i.e., it is generalizable, but has not yet been generalized) and then (2) the researcher, in examining it empirically, can demonstrate its generality.

Using the terms generality, generalization, generalize, general, generalizing, and generalizable, we now proceed to identify four types of generalizing.

\section{Four Types of Generalizing}

Our purpose in this study is to clarify the different processes of generalizing so that academic researchers in IS can better achieve and claim generality in their research. To this end, we offer a framework that draws attention to four types of generalizing.

First, we recognize that the act of generalizing is not something done only by academic researchers, but also something done by everyday people in everyday life. Consultants and managers, for instance, can (and, arguably, must) generalize from just one or two experiences. We therefore define one dimension of generalizing as referring to the context of inquiry. This context could be the inquiry of a person in everyday life (such as a consultant or a manager) or alternatively the inquiry of a person in academic

\footnotetext{
${ }^{2}$ We are aware of no published empirical study in the field of information systems that has ever offered explicit statements about what constitutes the entire class of empirical referents that its theory purports to explain. Even our own publications (i.e., the research that the authors of this paper have published) do not do this. It would be fair and justifiable to say that researchers generally imply what the class is (e.g., it could be "all managers" or "all corporations"). Still, we believe that, as a matter of good methodology, it would be a good idea for empirical studies to adopt this practice.
} 
Table 1. Four Types of Generalizing

\begin{tabular}{|cc|c|c|}
\cline { 3 - 4 } \multicolumn{1}{c|}{} & \multicolumn{2}{c|}{$\begin{array}{c}\text { Context } \\
\text { Inquiry in } \\
\text { Everyday Life }\end{array}$} & $\begin{array}{c}\text { Inquiry in } \\
\text { Academic Research }\end{array}$ \\
\hline \multirow{2}{*}{$\begin{array}{c}\text { Reasoning } \\
\text { Process }\end{array}$} & $\begin{array}{c}\text { everyday inductive } \\
\text { generalizing } \\
\text { Induction }\end{array}$ & $\begin{array}{c}\text { academic inductive } \\
\text { generalizing }\end{array}$ \\
& Deduction & "typification" & "generalizability" \\
& everyday deductive & academic deductive \\
generalizing & "learning" & "falsification" \\
\hline
\end{tabular}

research (such as an IS researcher). In the context of inquiry in everyday life, the generality that a person associates with a belief is largely determined by the social traditions of the person's group. In contrast, in the context of inquiry in academic research, the influence of social traditions of the academic group in establishing generality are strongly mediated by rigorous notions of evidence, logic, and methodology in "scientific" thinking. Second, we recognize two different conceptions of the process of generalizing. These are inductive generalizing (a reasoning process that begins with observations and subsequently uses them as the basis on which to build a theory) and deductive generalizing (a reasoning process that begins with a theory and subsequently processes or tests it against observations). In Table 1, we use these two dimensions to identify four types of generalizing. We will now examine each of the four types in greater detail. Each type will be illustrated by means of an example. The examples were chosen not only because these illustrate the principles, but also because there are published details that illuminate the generalization process in each example.

\subsection{Everyday Inductive Generalizing}

The sociologist and phenomenologist Schutz offers an explanation of how everyday people in everyday life make generalizations.

All projects of my forthcoming acts are based upon my knowledge at hand at the time of projecting. To this knowledge belongs my experience of previously performed acts which are typically similar to the projected one....The first action $\boldsymbol{A}^{\prime}$ started within a set of circumstances $C^{\prime}$ and indeed brought about the state of affairs $S$; the repeated action $\boldsymbol{A}$ "starts in a set of circumstances $\boldsymbol{C}$ " and is expected to bring about the state of affairs $S^{\prime \prime}$. [Schultz 1973, p. 20] 
Regarding a person's general conception of a type of action, Schutz acknowledges that no two real-world instances or instantiations of it (e.g., $\boldsymbol{A}^{\prime}$ and $\left.\boldsymbol{A}\right)$ ) are ever exactly the same, which leads to the following point regarding how a person in everyday life makes a generalization:

Yet exactly those features which make them unique and irretrievable in the strict sense are- to my common-sense thinking —eliminated as being irrelevant for my purpose at hand. When making the idealization of "I-can-do-it-again" I am merely interested in the typicality of $\boldsymbol{A}, \boldsymbol{C}$, and $\boldsymbol{S}$, all of them without primes. The construction consists, figuratively speaking, in the suppression of the primes as being irrelevant, and this, incidentally is characteristic of typifications of all kinds.

What Schutz calls a "typification" is further discussed by Berger and Luckmann in their classic book The Social Construction of Reality (1966). In one person's interactions with another person, the former could "apprehend the latter as 'a man,' 'a European,' 'a buyer,' 'a jovial type,' and so on" (Berger and Luckmann 1996, p. 31), where "these typifications ongoingly affect" how the former interacts with the latter. Typifications serve the purpose of allowing everyday people to negotiate their interactions with one another, as well as with the physical world around them. Returning to Schutz's example, we denote the process of generalizing in everyday life as follows:

$$
A^{\prime}, A^{\prime \prime}, A^{\prime \prime \prime}, \ldots===>A
$$

Another example, again referring to Schutz's typifications, would be:

$$
\left\{A^{\prime}, C^{\prime}, S\right\},\left\{A^{\prime \prime}, C^{\prime \prime}, S^{\prime \prime}\right\},\left\{A^{\prime \prime \prime}, C^{\prime \prime \prime}, S^{\prime \prime}\right\}, \ldots===>\{A, C, S\}
$$

The reasoning process that produces such typifications is what we call "everyday inductive generalizing." We describe this reasoning process as "everyday" because it refers to inquiry in everyday life, rather than inquiry in academic research. We identify this reasoning process to be "inductive generalizing" because it begins with observations and subsequently uses them as the basis on which to generalize and then construct the typification.

\section{Example of everyday inductive generalizing in IS}

For an example, we will focus on one particularly interesting typification constructed by the professionals who work in the everyday world of IS development. It is what they call the "death march project."

They have come to see the "death march project" in the following way. It involves IS projects where signs of failure are apparent, but all participants in the project nonetheless proceed to play their parts, as if there were nothing wrong. "Death march projects" have come to be known, at least to those in the everyday world of IS development, by certain notable signs. One sign is that the risk of project failure appears 
greater than 50\%. Other frequent signs of a "death march project" include irrational managerial compensation, the drowning of staff with a complete, sudden conversion to a new silver-bullet methodology, and the driving away of staff through cancellation of vacations and weekends (Yourdon 1997). Through everyday inductive generalizing across their observations of such IS development settings, software engineering practitioners have constructed the "death march project" typification. The sorts of projects now seen as "death march" projects are not new, having existed since the 1960s (Yourdon 1997); however, the typifying or generalizing of these observations into the shared, everyday concept of "death march" occurred only in the 1990s.

The process by which the "death march" typification results from the everyday inductive generalizing performed by a group of developers is illustrated in the February 1997 issue of American Programmer, which is dedicated to the topic of death march projects. In this issue, corporate IS practitioners and IS consultants speak normatively from their experiences with death march projects. Each death march project in their experience constitutes a discrete phenomenon that they have experienced, where $\boldsymbol{P}^{\prime}$ could be the SMS/800 nine hour project (Oxley and Curtis 1997) and $\boldsymbol{P}^{\prime \prime}$ could be the billing and accounts receivable system (Roberts 1997). Across these discrete experiences, they have generalized to $\boldsymbol{P}$, the death march project:

$$
\boldsymbol{P}^{\prime}, \boldsymbol{P}^{\prime \prime}, \boldsymbol{P}^{\prime \prime \prime}===>\boldsymbol{P}
$$

$\boldsymbol{P}$, the "death march project" typification, is the general case. The process of generalizing commences by noting characteristics shared across the observed cases $\boldsymbol{P}^{\prime}, \boldsymbol{P}^{\prime \prime}, \boldsymbol{P}^{\prime \prime \prime}$, etc. Examples of these shared characteristics are certain irrational management actions noted above. The existence of the "death march project" was never "hypothesized" or "tested" in the scientific sense of these terms. Rather, this general case is a consequence of the generalizing by everyday IS developers across certain unpleasant projects. The general case is significant to these developers because of its instrumentality. The developers use the concept as a normative model to suggest possible actions when they encounter death march projects in the future. Although not tested for the status of scientific truth, the "death march" typification acquires the status of everyday truth "in so far as [it] helps us to get into satisfactory relation with other parts of our experience" (James 1975, p. 35).

\subsection{Everyday Deductive Generalizing}

Not all generalizations in everyday life are the "outputs" of a reasoning process for which particular instances are the "inputs." In other situations, an already existing generalization is itself the "input" to a reasoning process that applies it, where the "output" is the result that the generalization suited, or failed to suit, the application.

Argyris and Schön (1978) describe the processes involved in testing extant general theories in everyday life for suitability in new applications. They distinguish between a person's "espoused theory" and the same person's "theory-in-use." A person's espoused theory is the explanation that this person would voice to explain her behavior. A person's theory-in-use is the theory that actually governs this person's behavior. The theory that a person espouses is not always the same as the person's theory-in-use; 
indeed, a person might not even be aware of her theory-in-use. When a person's actions, based on her theory-in-use, repeatedly elicit surprising reactions from other people or other parts of a person's environment-that is, when the theory-in-use that provided the basis for actions apparently does not work-an error or deficiency in the theory-in-use is sensed. Once this deficiency is sensed, the person can proceed to change her theoryin-use, her espoused theory, or both.

The everyday reasoning process in this behavior is not inductive, but deductive; it begins not with the observations of particulars, but with an already existing generalization (the person's theory-in-use). The person applies the generalization to a set of particulars she observes in the empirical setting in which she seeks to act (rather than derive the generalization from these particulars). Two results are possible. In one, the person notices surprises in the reactions to her actions, whereupon she learns that her theory-in-use needs to be changed (which would involve and require her becoming conscious of her theory-in-use for the first time). In the other result, there is nothing surprising for the person to notice, whereupon her theory-in-use survives and becomes, in a sense, further entrenched or stronger as a generalization. We refer to this reasoning process as "everyday deductive generalizing."

Everyday deductive generalizing is most apparent when conflict and "abnormal" discourse signal inconsistencies between the shared organizational espoused theories and the theories-in-use of individuals. In the process of this discourse, called "double-loop learning," the institutionalized generalizations are adjusted to match the individual experiences and theories-in-use. These corrected, espoused theories may then stand as general theories for further application.

Argyris and Schön were hardly the first scholars to recognize that deductive generalizing occurs in everyday life. For instance, this form of generalizing is the hallmark of the philosophy of pragmatism as found in the respective works of William James, John Dewey, and Charles Sanders Pierce.

\section{Example of everyday deductive generalizing in IS}

For an example, we will focus on the generalizing in which some people engaged in an IS action research project.

Action research is a research method that aims to solve immediate practical problems while expanding scientific knowledge. Based on collaboration between researchers and research subjects, action research is a cyclical process that builds learning about change in a given social system (Hult and Lennung 1980). Unlike laboratory experiments that isolate research subjects from the real world, action research involves intervention experiments in which the researchers, along with the research subjects, apply a stimulus or other change strategy to the real-world context in which the research subjects live or work. In action research, intervention experiments operate on problems or questions that the practitioners (to whom we also refer interchangeably as research subjects and everyday people) themselves perceive within the context of their own particular empirical setting. Participatory action research is distinguished by the additional characteristic of involvement of, first, practitioners not only as subjects but also as co-researchers and, second, researchers not only as scientists, but also as subjects. "It is based on the Lewinian proposition that causal inferences about the behavior of 
human beings are more likely to be valid and enactable when the human beings in question participate in building and testing them" (Argyris and Schön 1991, p. 86). Because of the deep collaboration between academics and everyday professionals, participatory action research involves both academic and everyday deductive generalizing. Consequently, examples of this form of research can illustrate the two categories of generalizing. However, in this portion of our study (section 2.2), we will focus on how the everyday professionals themselves engage in deductive generalizing. (We will defer our discussion on how the academic researchers engage in academic deductive generalizing until section 2.4.)

One example of an action research project in IS appears in Baskerville and Stage's study (1996) on risk management for prototyping. We may summarize their study as follows. It addresses one of the main problems in the overall practice of prototyping that, at least at the time of the study, had not yet been resolved. The practical problem was the difficulty simply in controlling the scope and unfolding development of prototyping projects. In general, this problem can severely limit the range of IS development projects in which prototyping can be used effectively. In Baskerville and Stage's study, the action research project developed and validated a new approach that uses an explicit risk mitigation model in the IS development process, one that focuses the collaborative action research team's attention on the consequences and priorities inherent in the prototyping situation. The study established that prototyping projects could be controlled if appropriate risk resolution strategies were put into effect prior to any breakdown in the prototyping process.

Baskerville and Stage's study illustrates how collaborative action team members engage in everyday deductive generalizing. In the example that follows, the generalizing pertains to the team members' original conception of a prototyping risk factor that they called "user alienation."

Specifically, while in the process of applying risk analysis as a tool to help administer their prototyping project, the collaborative action research team members stated certain expectations during an initial analysis of the risk factors that they perceived in the problem setting of their practical prototyping. Using the Argyris and Schön terminology that we introduced above, we can say that the collaborative action research team members came to "espouse" a theory that named 12 risk factors. At the same time, because they actually applied and followed their "espoused theory," we may state that, in this case, the "espoused theory" coincided with their "theory-in-use."

The collaborative action research team members stated one of the 12 risk factors as "Users will not understand what we are doing" (Baskerville and Stage 1996, p. 493) and they stated the consequence of this factor as "The users become alienated." They ranked this factor's risk level as moderate.

However, during the first prototyping cycle, the participants noticed that their understanding of risk factors led to a surprising result. The participants noted the surprise that the mentioned risk factor carried not just one, but actually two separate consequences. The first of the two separate consequences was enduring and serious: "The users do not know what product they will receive" (p. 495). The second was transient and trivial: "The users do not understand their role in the development process." The need to differentiate the original single consequence into two became obvious during the first learning cycle as the users gained experience with prototyping 
and adapted enthusiastically to their role in the development process (Baskerville and Stage 1991, p. 12).

To recapitulate, we note that the intervention experiment was not inductive, but deductive; the intervention experiment began not with the observations of particulars, but with a generalization about risk factors that the collaborative research action team members had previously formulated. As required in the invention phase of action research, the researchers were not observing as detached scientists, but instead were participating in the role of everyday people themselves who are acting and thinking in the real world. In their intervention, all of the team members applied their already existing generalization to a set of particulars that they observed in the IS development context in which they sought to act. In the results from their intervention, they noticed the major surprise in which there was not just one but two different consequences to one of their theorized risk factors, whereupon they learned that their understanding needed to be changed. We refer to this reasoning process as an instance of everyday deductive generalizing. Moreover, in this particular instance, the conclusion was not to claim greater generality for their initial understanding, but rather, to establish that it lacked generality. The everyday people learned that they needed to change their already existing generalization. ${ }^{3}$

\subsection{Academic Inductive Generalizing}

We observe that, among many (and sometimes, it seems like most) IS researchers, there is the (mis)conception that the greater the sample size, number of observations, or other quantity of empirical material in a research study, then the more "generalizable" (or, in the terminology of our study, the more general) the resulting theory is. We use the term "academic inductive generalizing" to refer to the process by which social scientific researchers would begin with observations (such as "n" sample points) and end up with a theory.

Lee (1999) reviews arguments, from the philosophy of science, that induction is fine for suggesting the formulation of a theory, but also that induction offers no help in testing or otherwise empirically validating a theory. The gist of his review is that (1) according to induction, the greater " $n$ " is (where " $n$ " is the number of observations across which a researcher would be generalizing a theory), the more general or "generalizable" the resulting theory is, but (2) the methodological principle that this procedure assumes - namely, that "inductive inference leads to valid theories"-is itself not empirically justifiably because (3) any attempt to provide an empirical justification of the statement, "inductive inference leads to empirically valid theories," would ultimately apply induction itself, thereby leading to an infinite regress in reasoning. ${ }^{4}$ Lee

\footnotetext{
${ }^{3}$ Using the Schutz-based terminology of $\{A, C, S\}$, the conclusion was that $\{A, C, S\}$ lacked generality. Specifically, $S$ did not fit and it needed to be replaced.

${ }^{4}$ Using the Schutz-based terminology, induction maintains that the greater the number of observations $\left(\boldsymbol{O}, \boldsymbol{O}^{\prime \prime}, \boldsymbol{O}^{\prime \prime \prime}\right.$, etc.), then the more general or "generalizable" the resulting theory, $\boldsymbol{T}$. In other words, in the following,

$$
O^{\prime}, O^{\prime \prime}, O^{\prime \prime \prime}, O^{\prime \prime \prime}, \ldots, O^{\prime \prime \prime . .}===>T
$$
}


notes that even the discipline of statistical inference has distanced itself from the notion of induction, where statistical inference recognizes that a larger sample size, "n," does not increase the probability that a statistically inferred proposition (such as a confidence interval) is true, but instead serves to enhance the "level of confidence" or "statistical significance," which is an attribute describing the researcher's investigation, not the truth or falsity of the proposition.

As we will explain in section 2.4 , deductive generalizing is the viable alternative to inductive generalizing in academic research.

\section{Examples of academic inductive generalizing in IS}

Although inductive generalizing is not a valid scientific procedure, many IS researchers hold themselves to it as the standard for establishing whether or not their research possesses what they call "generalizability" (or what we call generality). And almost always, they apply this standard (which they do not know to be incorrect) so as to conclude (incorrectly) that their research lacks "generalizability" (generality). We find instances of this when we look at IS researchers who conduct case studies. They regard the single case or small number of cases that they examined to dampen the "generalizability" (generality) of their results. Applying the same logic, they say that "generalizability" (generality) can result only by increasing "n."

From the point of view of theory development, while case studies provide useful anecdotal information, the generalizability from one specific instance to another is often limited. [Albers, Agarwal and Tanniru 1994, p. 94]

First and foremost, it should be reaffirmed that the single case research strategy employed here only allows generalizability to a research model, which in turn needs to be tested under a multiple case study design or by other field methods. [Brown 1997, pp. 90]

From the evidence of the two cases, it was not possible to identify any generalisable [sic] strategies for overcoming constraints but the particular solutions developed appeared to reflect the developers'

the presumption is that as the number of observations (the instances on the left hand side of the arrow) increases, then the "generalizability" (generality) of the theory (on the right hand side of the arrow) also increases. The flaw is that the presumed statement, "as the number of observations increases, then the 'generalizability' (generality) of the theory also increases," is itself not empirically justifiable. For this insight, Lee credits Popper (1968) who, in turn, cites the philosopher Hume.

A not insignificant, additional point is that the presumed statement, "as the number of observations increases, then the 'generalizability' (generality) of the theory also increases," also begs the question of how it is possible to derive theoretical statements comprising $T$ (which typically posit the existence of unobservable entities and relationships) from observation statements. If the many observations $\boldsymbol{O}^{\prime}, \boldsymbol{O}^{\prime \prime}, \boldsymbol{O}^{\prime \prime \prime}, \boldsymbol{O}^{\prime \prime \prime \prime}, \ldots, \boldsymbol{O}^{\prime \prime \prime \prime .}$ can be generalized to anything, they would be generalized to $\boldsymbol{O}$,

$$
\boldsymbol{O}^{\prime}, \boldsymbol{O}^{\prime \prime}, \boldsymbol{O}^{\prime \prime \prime}, \boldsymbol{O}^{\prime \prime \prime}, \ldots, \boldsymbol{O}^{\prime \prime \prime . .}==\Rightarrow \boldsymbol{O}
$$

rather than to $\boldsymbol{T}$. This amounts to another flaw in the logic of induction regarding the empirical justification of a theory. 
local conditions and their knowledge, intuition, and experience. This would suggest that rather than giving a set of generalized guidelines for improving user involvement (as is common in the literature), the emphasis might be better placed on supporting developers' ingenuity and improvisation and on developing their social skills to enable them to overcome the constraints on involvement. [Nandhakumar and Jones 1997, p. 84]

Because they are drawn from a study of two organizations, these results should not be generalized to other contexts. Each context is different, so we should expect different contextual elements to interact with technical initiatives to produce different consequences. The findings should not even be extended to other settings where GIS, or even Arc/Info, is implemented. What is true for GIS in the two local county governments studied may be untrue for GIS in other governmental units or in private enterprises. [Robey and Sahay 1996, p. 108]

In particular, in-depth analysis of extensive data from only one organization reduces generalizability, but increases correspondence to reality. [Hidding 1998, p. 311]

The study has a number [of] limitations that need to be considered in making any conclusions. First, the single case site limits the generalizability of results. The purpose of the study was not to provide generalizability of empirical results to other firms, rather the purpose was to "expand and generalize theories" (Yin 1984). [Jarvenpaa and Leidner 1997, p. 408]

Conducting additional case studies will provide instances of the various learning/outcome combinations, and we encourage such research. On the other hand, case studies alone will not result in validity or generalizability. Toward that end, a more fruitful approach might be to compare the development processes for similar systems in different organizations, or two or more systems being developed in a single organization. [Stein and Vandenbosch 1996]

The irony is that these IS researchers' method-in-use (with respect to generalizing) derives from a naïve and invalid statistical notion-the notion that a larger sample size leads to greater "generalizability" (generality) — to which even the field of statistics itself does not subscribe. However, once we understand the invalidity of this notion, we notice that the generality of the reported cases studies is inherently no different from the generality of statistically conducted, large-sample studies. As for IS researchers who flagellate themselves for a supposed lack of "generalizability" (generality) in their case studies, we believe that they are not only doing this unnecessarily, but also harming the overall reputation of the academic discipline of IS. This reputation is diminished in two ways: first, by reinforcing and perpetuating an incorrect notion of science (i.e., validity is achieved through induction) and, second, demeaning (and thereby discouraging the 
dissemination of) their own research findings whenever they draw public attention to a flaw that does not really exist.

\subsection{Academic Deductive Generalizing}

Lee (1999) also reviews arguments that explain how deductive inference operates in science. He notes that (1) the statements comprising a theory typically posit the existence of unobservable entities, such as molecules, atoms, electrons, and protons (hence making it appropriate for these statements to be called "theoretical"), (2) a researcher applies the theoretical statements to the facts describing a specific situation (such as the initial conditions in an experiment), allowing the deductions of predictions of what the researcher subsequently ought to observe or not to observe if the theory is true, upon the application of the experimental stimulus or treatment, and (3) the researcher, in comparing what the theory predicts in this specific situation and what she actually observes in this specific situation, ends up testing the theory deductively and indirectly. ${ }^{5}$ If the prediction turns out to be false, then the theory from which it followed deductively would be false as well. On the other hand, if the prediction turns out to be true, then "the truth of the theory (from which the prediction originated) is not proven, but is only 'corroborated,' 'supported,' or 'confirmed' in the instance of this single test" because other new instances would open up the same theory to yet new opportunities for its falsification. Hence, in the deductive logic of empirical science, a theory can never be definitively shown to be true, but must remain forever falsifiable. Lee states, "the widespread characterization of theories, even in the social sciences, as falsifiable, testable, refutable or disconfirmable [is] an indication of the widespread extent to which the deductive testing of theories is practiced."

For academic deductive generalizing, unlike academic inductive generalizing, a larger number of observations consistent with the theory being studied does not increase the generality attributable to the theory. For instance, to increase the number of observations consistent with Newtonian physics is indeed possible, but hardly makes Newtonian physics more general (indeed, it is false and has been superseded by Einsteinian physics). Likewise, to increase the number of observations consistent with Ptolemaic astronomy is indeed possible, but hardly makes the theory of an earth-centered solar system more general! Therefore, in academic deductive generalizing, additional observations (such as a larger sample size) supportive of a theory do not render the theory any more general or true. This also means that the only observations that a deductive scientific researcher may regard as contributing useful information in gauging whether a theory is true or false are those that contradict the theory; such empirical

\footnotetext{
${ }^{5}$ Using the Schutz-based terminology, instead of having $\left\{A^{\prime}, C^{\prime}, S\right\}$, where $A^{\prime}$ refers to an instantiated action, $C^{\prime}$ 'refers to an instantiated set of circumstances, and $S^{\prime}$ refers to an instantiated state of affairs that $A^{\prime}$ engenders in $C^{\prime}$, we have $\left\{\boldsymbol{T}, \boldsymbol{C}^{\prime}, \boldsymbol{S}_{\boldsymbol{P}}{ }^{\prime}, \boldsymbol{S}_{\boldsymbol{O}}{ }^{\prime}\right\}$, where $\boldsymbol{T}$ refers to the scientist's theory, $\boldsymbol{C}^{\prime}$ refers to the specific empirical conditions (the "initial conditions") instantiated in an experiment, $S_{P}$ 'refers to the specific state of affairs expected and predicted (hence the subscript ${ }_{P}$ in $S_{P}$ ) to follow in the experiment if the theory is true, and $S_{o}$ 'refers to the specific state of affairs actually observed (hence the subscript ${ }_{o}$ in $S_{o}$ ) to follow in the experiment. The theory $\boldsymbol{T}$ is falsified if $S_{P}{ }^{\prime}$ and $\boldsymbol{S}_{o}{ }^{\prime}$ differ. The theory $\mathbf{T}$ is corroborated or confirmed if $\boldsymbol{S}_{\boldsymbol{O}}{ }^{\prime}$ matches $S_{P}$ ', but remains falsifiable in future experiments involving the new circumstances, $C^{\prime \prime}$, and then $C^{\prime \prime \prime}$, and then $C^{\prime \prime \prime \prime}$, and so on.
} 
evidence would, at best, throw a theory's validity and generality in doubt and, at worst, result in the researcher's conclusion that the theory is false and hence possesses no generality.

\section{Example of academic deductive generalizing in IS}

For an illustration of academic deductive generalizing, we return to the Baskerville and Stage action research study (1996) that we introduced in section 2.2. The example involves the detection of an important delusory element in the researchers' risk analysis. This element materialized when a risk factor, although highly expected, failed to develop as time progressed. The risk factor, "The technical environment is unreliable" (p. 493), was included because the prototyping project was forced to use a recent release of a database software package known sometimes to corrupt database files.

The software performed flawlessly in the early stages of the prototype construction. Based on this experience, the members of the collaborative action research team downgraded the probability of the risk factor in question. In this scenario, we can see that the researchers posited a hypothesis ("this software is unreliable") and conducted an experiment (prototype construction) that failed to support the hypothesis-hence (deceptively) encouraging them to attribute less generality to it. However, as the prototype development continued, its functionality and complexity rose. With this intensified usage, many previously unused features of the application generator were called into operation, with the result that the database software finally corrupted a table. This example thus demonstrates the action research equivalent to a Type II error, imputing support for a null hypothesis from the failure of experience to support the alternative hypothesis.

Since the time of their study's being published, the researchers have felt confidence in being able to apply their lesson beyond the particular action-research case is which it arose. They have formulated a general case in which prototyping management teams are inclined to reduce the probability rank of a known risk factor while the conditions that lead to such a risk became less favorable. Teams, in general, are theorized to rely on their own experience with risky aspects of projects, revealing a "guardian angel" mentality that inclines teams to ignore severely rising risk. The generality and confidence associated with this revised theory will remain ungauged until tested, like any other scientific theory.

\section{Discussion}

What is an appropriate way in which information systems researchers may lay claim to generality for their research?

First, and most important, information systems researchers should not give up claims to generality on the basis that their research involves a small " $n$ " (for instance, a small number of organizations observed). The reason is that this would presume and reinforce the fallacy of the logic of induction-a logic rejected even by the naturalscience model of research. A natural scientist who draws conclusions from an experiment does not rush to disclaim generality of her results on the basis that they are 
based on only a "single" experiment. In the same way, an information systems researcher who draws conclusions from a single field site need not rush to disclaim the generality of his results.

Second, the analogy to the experimental natural scientist also reveals how an information systems researcher may indeed lay claim to generality in her study. A natural scientist performing an experiment with favorable results (i.e., the theory's predictions are corroborated, not refuted) would need to state the details of the particular empirical circumstances for which the observed experimental results occurred. A claim of generality for the theory would mean that the theory can be expected also to hold in other instances that share the same empirical circumstances. ${ }^{6}$ We note that an information systems researcher who conducts a case study (or other intensive research) would be as able to do this as a natural scientist who conducts an experiment. And just as the natural scientist conducting an experiment would not need to apologize for her theory's not necessarily applying to empirical circumstances different from those in her experiment, the information systems researcher who conducts a case study (or other intensive research) would not need to apologize for his theory's not necessarily applying to empirical circumstances different from those in his field site. Along the same lines, future efforts to enhance the generality of the information systems researcher's theory would not involve indiscriminately and randomly collecting more observations, but rather, would involve the targeting of additional specific empirical conditions to which the applicability of the theory is being questioned and, pending the observational results, would or would not be extended.

Returning to some of the published examples of empirical research in the information systems field that we presented in section 2.3, we suggest the following. We quoted Albers, Agarwal and Tanniru (1994, p. 94) as saying: "From the point of view of theory development, while case studies provide useful anecdotal information, the generalizability from one specific instance to another is often limited." Our suggestion is that Albers, Agarwal, and Tanniru can say instead: "From the point of view of theory development, a case study should not be characterized as providing anecdotal information, but instead should be valued, first, for concretely demonstrating a specific instantiation of the circumstances in which the developed theory is known to apply and, second, for allowing additional applications of the same theory to other situations also involving instantiations of the same circumstances. Specifically, these circumstances are...." Similarly, we quoted Robey and Sahay (1996, p. 108) as saying: "Because they are drawn from a study of two organizations, these results should not be generalized to other contexts. Each context is different, so we should expect different contextual elements to interact with technical initiatives to produce different consequences." Our suggestion is that Robey and Sahay can say instead: "Because they are drawn from a study of two organizations, these results can apply to other contexts sharing the same circumstances of these two organizations. Where the elements in other contexts are the same in these two organizations, we can expect interactions of these elements with technical initiatives to produce the same consequences. Specifically, these circum-

\footnotetext{
${ }^{6}$ Using the Schutz-based terminology, suppose we have $\left\{T, C^{\prime}, S_{P}{ }^{\prime}, S_{o}{ }^{\prime}\right\}$, as we defined in the previous footnote. In the event that $S_{o}$ 'matches $S_{P}$, we can say that the theory $T$ is sufficiently general for us to expect it to apply in all future occasions (whether future experimental settings or future organizational settings) that instantiate the set of circumstances $C^{\prime}$.
} 
stances are...." Finally, we quoted Hidding (1998, p. 311) as saying: "In particular, indepth analysis of extensive data from only one organization reduces generalizability...." Our suggestion is that Hidding can say instead: "In particular, in-depth analysis from one organization enriches our knowledge of the fine details of the empirical circumstances in which the theory applies, hence increasing the reliability with which we can apply this theory in new settings in future occasions. Specifically, we can report the fine details of the empirical circumstances as comprising...."

Third, this notion of how to lay claim to generality also has an obvious ramification for practice. When an information systems researcher demonstrates that her theory applies in certain circumstances, a practitioner would know that these are the circumstances in which the theory could be used. ${ }^{7}$ Where information systems researchers simply abandon claims of generality and hence forego specifying what these circumstances are, the transfer of academic findings to professional practice would be aborted.

\section{Conclusion}

The existing concept of "generalizability" is fastened on one peculiar form of generalizing. The concept is further confounded in the IS literature by its conflation with distinct concepts like generality and generalizing. Untangling this confusion reveals a variety of obscured, and sometimes legitimate forms, of generalizing. Many intensive research ventures could satisfy one of these forms, yet the authors selfflagellate over their inability to found their generalities on the one peculiar form that IS researchers have mindlessly idealized.

The self-flagellation is not merely annoying; it is harmful. When researchers unnecessarily divest their right to claim generality, their research audience is denied their analysis of the utility of their theories. When researchers generalize and claim generality, they encompass the larger scope of phenomena, beyond those directly captured by their research, to which their findings and understandings apply (Babbie 1990). By renouncing their right to generalize and claim generality, intensive researchers lose the latitude to explain the wide field of uses for their findings.

The IS research field is relatively vocational and operates in concert with technologies that are incredibly fast-moving. Our understanding of the social and organizational aspects of our field may be trailing far behind our grasp of the technical issues. Unnecessarily confining the application of new theories from intensive research is helping to cripple our ability to keep pace. Applying a variety of forms of generalizing in information systems promises to improve the development of more current and more useful social and organizational theory.

${ }^{7}$ Using the Schutz-based terminology, the same circumstances would be the $C^{\prime}$ in $\left\{T, C^{\prime}, S_{P}{ }^{\prime}, S_{o}{ }^{\prime}\right\}$ for the information systems researcher engaging in academic generalizing, and the $C^{\prime}$ in $\{A, C, S\}$ for the practitioner engaging in everyday generalizing. 


\section{References}

Albers, M.; Agarwal, R.; and Tanniru, M. "The Practice of Business Process Reengineering: Radical Planning and Incremental Implementation in an IS Organization," in SIGCPR '94. Proceedings of the 1994 Computer Personnel Research Conference on Reinventing IS: Managing Information Technology in Changing Organizations. Arlington, VA: ACM Press, 1994, pp. 87-96.

Argyris, C., and Schön, D. Organizational Learning: A Theory of Action Perspective. Reading, MA: Addison-Wesley, 1978.

Argyris, C., and Schön, D. "Participatory Action Research and Action Science Compared" in Participatory Action Research, W. F. Whyte (ed.). Newbury Park, NJ: Sage, 1991, pp. 8596.

Babbie, E. Survey Research Methods, $2^{\text {nd }}$ ed. Belmont, CA: Wadsworth, 1990.

Baskerville, R. L., and Stage, J. "Developing the Prototype Approach in Rapid Systems Modelling." Working Paper, Institute for Electonic Systems R91-35, The University of Aalborg, Aalbor, Denmark, September 1991.

Baskerville, R., and Stage, J. "Controlling Prototype Development Through Risk Analysis," MIS Quarterly (20:4), 1996, pp. 481-504.

Berger, P., and Luckmann, T. The Social Construction of Reality: A Treatise in the Sociology of Knowledge. New York: Anchor Press, 1966.

Brown, C. "Examining the Emergence of Hybrid IS Governance Solutions: Evidence from a Single Case Site," Information Systems Research (8:1), 1997, pp. 69-94.

Hidding, G. J. "Adoption of IS Development Methods Across Cultural Boundaries," in Proceedings of the Nineteenth International Conference on Information Systems, R. Hirschheim, M. Newman, and J. I. DeGross, (eds.), Helsinki, Finland, 1998, pp. 308-312.

Hult, M., and Lennung, S. “Towards a Definition of Action Research: A Note and Bibliography," Journal of Management Studies (17), 1980, pp. 241-250.

James, W. "What Pragmatism Means," in Pragmatism, W. James ( ed.). Cambridge, MA: Harvard University Press, 1975, pp. 27-44.

Jarvenpaa, S. L., and Leidner, D. E. "An Information Company in Mexico: Extending the Resource-based View of the Firm," in Proceedings of the Eighteenth International Conference on Information Systems, K. Kumar and J. I. DeGross (eds.), Atlanta, GA, 1997, pp. 75-87.

Lee, A. S. “A Scientific Methodology for MIS Case Studies,” MIS Quarterly (13:1), 1989, pp. 33 50.

Lee, A. S. "Researching MIS," in Rethinking Management Information Systems: An Interdisciplinary Perspective, W. L. Currie and R. D. Galliers (eds.). London: Oxford University Press, 1999.

Nandhakumar, J., and Jones, M. "Designing in the Dark: The Changing User-developer Relationship in Information Systems Development," in Proceedings of the Eighteenth International Conference on Information Systems, K. Kumar and J. I. DeGross (eds.), Atlanta, GA, 1997, pp. 75-87.

Oxley, D., and Curtis, B. "Reliable Work on a Death March Schedule," American Programmer (10:2), 1997, pp. 12-15.

Popper, K. R. The Logic of Scientific Discovery. New York: Harper Torchbooks, 1968.

Roberts, S. M. "Surviving and Succeeding in a Death March Project," American Programmer (10:2), 1997, pp. 12-15.

Robey, D., and Sahay, S. “Transforming Work Through Information Technology: A Comparative Case Study of Geographic Information Systems in County Government," Information Systems Research (7:1), 1996, pp. 93-110.

Schutz, A. "Common Sense and Scientific Interpretation of Human Action," in Alfred Schutz, Collected Papers I: The Problem of Social Reality. The Hague: Martinus Nijhoff, 1973. 
Stein, E. W., and Vandenbosch, B. "Organizational Learning During Advanced System Development: Opportunities and Obstacles," Journal of Management Information Systems (13:2), 1996.

Yourdon, E. "Editorial.” American Programmer (10:2), 1997, p. i-1.

\section{About the Authors}

Richard Baskerville is an Associate Professor in the Department of Computer Information Systems at Georgia State University. His research focuses on security and methods in information systems, their interaction with organizations and research methods. He is an associate editor of The Information Systems Journal and MIS Quarterly. Richard's practical and consulting experience includes advanced information system designs for the U.S. Defense and Energy Departments. He is chair of the IFIP Working Group 8.2, and a Chartered Engineer under the British Engineering Council. Richard holds M.Sc. and Ph.D. degrees from the London School of Economics. He can be reached at baskerville@acm.org.

Allen S. Lee is a Professor in the Department of Information Systems at Virginia Commonwealth University and the Eminent Scholar of the Information Systems Research Institute. He has been a proponent of qualitative, interpretive, and case research in the study of information technology in organizations. He is Editor-in-Chief of MIS Quarterly and co-editor of the book, Information Systems and Qualitative Research (London: Chapman \& Hall, 1997). Allen can be reached by e-mail at AllenSLee@csi.com. 